\title{
Isolated intracranial hypertension following SARS-CoV-2 infection in an adolescent boy: a case report
}

\author{
Alaa ELmazny ${ }^{1,2} \cdot$ Shireen Mostafa Shousha ${ }^{3} \cdot$ Samira Saraya $^{4} \cdot$ Rehab Magdy $^{1}(\mathbb{C}$
}

Received: 19 July 2021 / Accepted: 24 September 2021 / Published online: 11 October 2021

(c) Belgian Neurological Society 2021

\section{Dear Editor,}

Idiopathic intracranial hypertension is a clinical condition characterized by an elevated cerebrospinal fluid pressure without any recognizable cause. The disease incidence is much higher among overweight or obese women in childbearing age [1]. Seven patients were previously diagnosed with intracranial hypertension during the course of severe acute respiratory syndrome coronavirus 2 (SARS-CoV-2) infection [2] and only one obese female patient of childbearing age was reported after recovery [3].

Herein, we describe a case of isolated intracranial hypertension in an adolescent, non-obese, male one week after recovering from SARS-CoV-2 infection.

\section{Case report}

A previously healthy 14 -year-old boy with a body mass index (BMI) of 21, presented to Neurology outpatient clinic at Kasr Al-Ainy hospital with a severe dull aching, holocephalic headache and blurring of vision of one-week duration not accompanied by nausea, vomiting, tinnitus, or transient visual obscurations.

Those symptoms developed 3 weeks after the patient tested positive for COVID-19 confirmed by a nasopharyngeal swab Real-Time PCR. He was diagnosed after developing loss of taste and smell, low- to moderate-grade fever and

Rehab Magdy

rehab.m.hassan@kasralainy.edu.eg

1 Department of Neurology, Kasr Al-Ainy Faculty of Medicine, Cairo University, Cairo, Egypt

2 Internal Medicine Department, Arabian Gulf University, Manama, Bahrain

3 Ophthalmology Department, Cairo University, Cairo, Egypt

4 Radiology Department, Kasr Al-Ainy Faculty of Medicine, Cairo University, Cairo, Egypt dry cough. However, he denied having headaches then. The patient received only supportive care at home and was not given any antibiotics, steroids, or anticoagulants. He recovered and was symptom-free within approximately 7 days.

The patient's general medical and neurological examinations were normal apart from bilateral optic disk swelling (grade II papilledema) detected using direct ophthalmoscopy.

Radiological assessment, including magnetic resonance imaging (MRI) and magnetic resonance venography (MRV), is illustrated in Fig. 1. Lumbar puncture was carried out under complete aseptic conditions in the lateral decubitus position. The opening pressure was $520 \mathrm{~mm} \mathrm{H}_{2} \mathrm{O}$, and cerebrospinal fluid (CSF) sample was taken for chemical analysis (protein level, glucose level, and cell count). Laboratory tests including complete blood picture, liver and kidney function tests, serum electrolytes, thyroid-stimulating hormone (TSH), autoimmune and coagulation profiles were also done to rule out secondary causes of intracranial hypertension. All blood tests and CSF analysis results came within normal ranges.

The carbonic anhydrase inhibitor, acetazolamide, was prescribed to the patient at a dose of $250 \mathrm{mg}$ every $6 \mathrm{~h}$ and stepped up after one week to $500 \mathrm{mg}$ tid, after which the patient reported total resolution of his symptoms and at 5 weeks of follow-up, the patient's papilledema grade improved from grade II to grade I. Automated perimetry (Humphrey field analyzer HFA II 750, USA) done at baseline and after 5 weeks is outlined in Fig. 2.

\section{Discussion}

The pathogenic mechanism underlying idiopathic intracranial hypertension remains unclear, but the proposed mechanisms include abnormalities in cerebral venous outflow, alterations in absorption or production of CSF, low-grade inflammation, and intracranial vascular clotting [1]. 


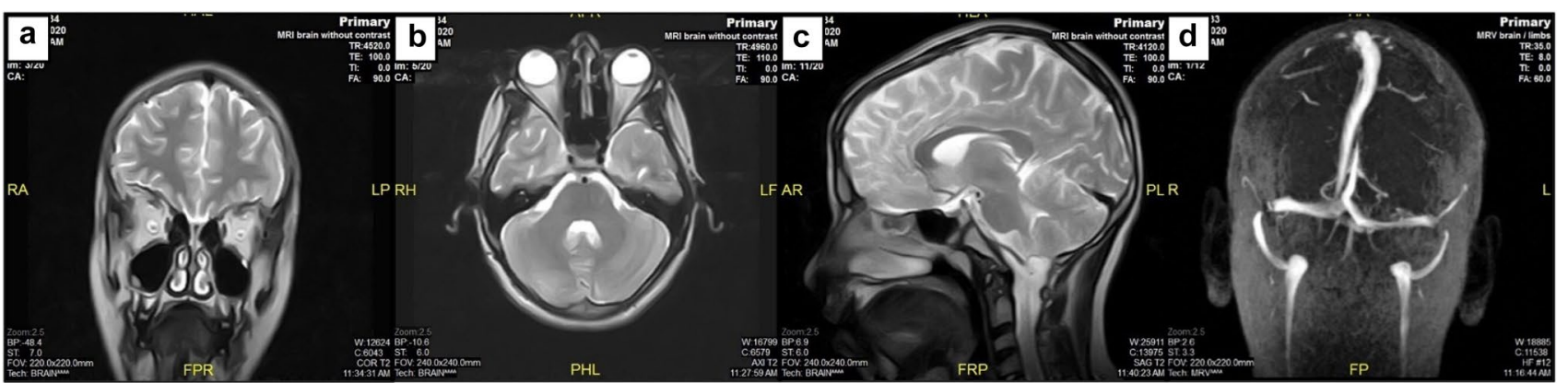

Fig. 1 a MRI T2-weighted coronal imaging showing increased fluid in the optic nerve sheath complex bilaterally. b MRI T1-weighted axial image showing flattening of the posterior globes and dilated

In this clinical scenario, a teenage boy had a headache and papilledema after recovery from SARS-CoV-2 infection. All his blood and CSF analysis results were unremarkable. To our knowledge, this is the first isolated intracranial hypertension case reported after recovery from SARS-CoV-2 infection in a non-obese adolescent boy.

SARS-CoV-2 may access the CNS through different hypothesized mechanisms. The main theory is retrograde axonal transport via sensory nerve endings embedded in the olfactory region. Another possible way is through angiotensin-converting enzyme 2 (ACE2) expressed in vascular endothelial cells, which mediates virus entry across the blood-brain barrier (BBB) [4].

How COVID-19 infection can precipitate isolated intracranial hypertension is a matter of discussion. Silva et al. [2] speculated that low-grade inflammation together with the hypercoagulable state attributed to SARS-CoV-2 infection could result in lower CSF absorption. On the other hand, Wostyn et al. [5] reported that SARS-CoV-2 optic nerve sheaths (c) MRI T1-weighted sagittal image showing a partially empty sella. d Normal magnetic resonance venography

infection might contribute to decreased CSF drainage via the cribriform plate and nasal mucosa as a result of olfactory epithelium destruction and olfactory sensory neurons loss which could also be a credible mechanism of anosmia in infected individuals [6]. The latter view could be a reliable mechanism of intracranial hypertension in our case.

Some drawbacks in this case report are noteworthy. First, SARS-CoV-2 RNA was not tested in the CSF. Second, longterm follow-up of the patient was not done.

\section{Conclusion}

This case scenario implies that SARS-CoV-2 infection could be a precipitating factor for isolated intracranial hypertension. Severe headache in subjects who have recovered from SARS-CoV-2 infection prompts investigational work-up for intracranial hypertension. 

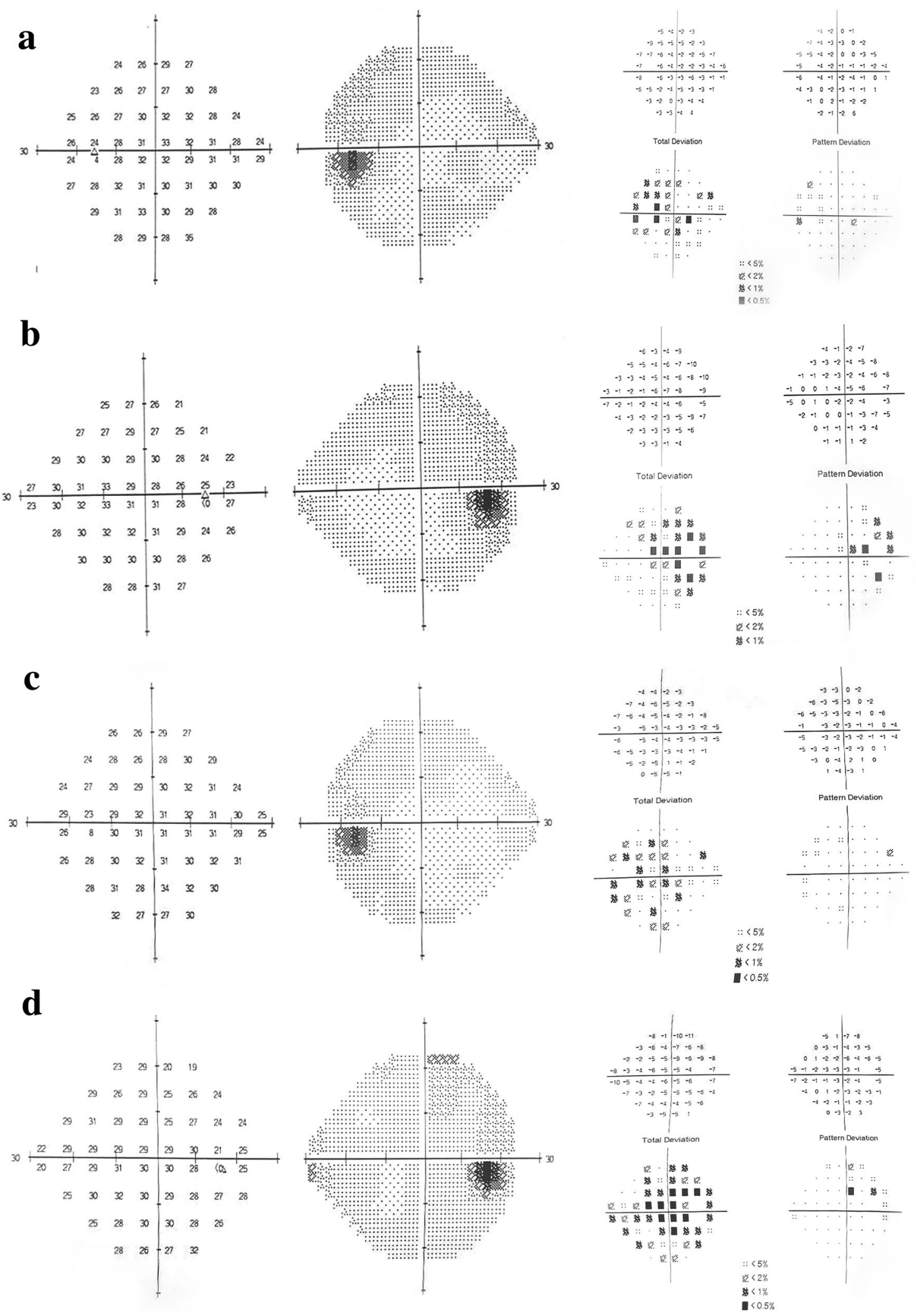

Fig. 2 Comparative automated perimetry SITA-standard for (a) Left eye at the first visit (b) Right eye at the first visit (c) Left eye after 5 weeks (d) Right eye after 5 weeks. Blind spot enlargement was noted in the first visit, which improved after 5 weeks for both eyes *Field reliability was accepted 


\section{Declaration}

Conflict of interest The authors declare that they have no conflict of interest.

Ethical approval All procedures performed in studies involving human participants were in accordance with the ethical standards of the institutional and/or national research committee and with the 1964 Helsinki declaration and its later amendments or comparable ethical standards.

Informed consent The authors certify that they have obtained an appropriate consent form from the patient's parent.

\section{References}

1. Mollan SP, Ali F, Hassan-Smith G, Botfield H, Friedman DI, Sinclair AJ (2016) Evolving evidence in adult idiopathic intracranial hypertension: pathophysiology and management. J Neurol Neurosurg Psyc 87:982

2. Silva MTT, Lima MA, Torezani G, Soares CN, Dantas C, Brandão $\mathrm{CO}$ et al (2020) Isolated intracranial hypertension associated with COVID-19. Cephalalgia Internat J Headache 40:1452-1458

3. Ilhan B, Cokal BG, Mungan Y (2021) Intracranial hypertension and visual loss following COVID-19: a case report. Indian J Ophthalmol.

4. Generoso JS, Barichello de Quevedo JL, Cattani M, Lodetti BF, Sousa L, Collodel A (2021) Neurobiology of COVID-19: how can the virus affect the brain?

5. Wostyn $\mathrm{P}$ (2021) COVID-19 and chronic fatigue syndrome: is the worst yet to come? Med Hypotheses 146:110469

6. Butowt R, von Bartheld CS (2020) Anosmia in COVID-19: Underlying mechanisms and assessment of an olfactory route to brain infection. Neuroscientist. 1073858420956905

Publisher's Note Springer Nature remains neutral with regard to jurisdictional claims in published maps and institutional affiliations. 